\title{
Atendimento odontológico as gestantes: revisão de literatura
}

\author{
Dental care for pregnant women: review literature \\ Atención dental para las mujeres embarazadas: revisión de la literatura
}

Hana Yasmim Marques Silva de Souza ORCID: https://orcid.org/0000-0003-0128-7720 Centro Universitário Tiradentes de Pernambuco, Brasil E-mail: hanayasmim@hotmail.com

Anne Caroline Silva Freire de Sá ORCID: https://orcid.org/0000-0003-3860-1373 Centro Universitário Tabosa de Almeida, Brasil E-mail:annecarolinefreiree@gmail.com

Brenda Graziella Coêlho Nogueira ORCID: https://orcid.org/0000-0003-0794-607X

Faculdade de Odontologia do Recife, Brasil E-mail: graziellanogueiraa@gmail.com

Daniele Saara dos Santos

ORCID: https://orcid.org/0000-0001-9381-5580

Universidade Federal de Pernambuco, Brasil E-mail: saaaradaniele@gmail.com

Edyssa Laryssa da Silva Ferreira de Araújo ORCID: https://orcid.org/0000-0002-4799-6330 Universidade Federal de Alagoas, Brasil E-mail: edyssa.araujo@foufal.ufal.br

George Jose de Oliveira Filho

ORCID: https://orcid.org/0000-0001-7275-9612 Centro Universitário Tiradentes de Pernambuco, Brasil E-mail: georgejose49@hotmail.com Ivanise Alencar de Sá

ORCID: https://orcid.org/0000-0003-1805-8393

Faculdade de Odontologia do Recife, Brasil

E-mail: ivanisealencarodonto@gmail.com

João Wictor Leandro da Silva

ORCID: https://orcid.org/0000-0002-9941-147X Centro Universitário Tiradentes de Pernambuco, Brasil E-mail: joao.wictor@souunit.com.br

Lisandre Letícia Gomes da Silva

ORCID: https://orcid.org/0000-0003-4338-1598 Centro Universitário Tiradentes de Pernambuco, Brasil

E-mail:lisandre_leticia2011@hotmail.com

Marcela Nogueira dos Santos

ORCID: https://orcid.org/0000-0002-0554-6382

Faculdade de Odontologia do Recife, Brasil

E-mail: marcelaanogueira.santos@gmail.com

Ranielly de Souza Silva

ORCID: https://orcid.org/0000-0002-5844-7564

Centro Universitário Tiradentes de Pernambuco, Brasil

E-mail: ranielly.souza@souunit.com.br

Thalita Oliveira da Silva Borba

ORCID: https://orcid.org/0000-0001-5539-7744

Centro Universitário Tabosa de Almeida, Brasil E-mail: Thalita.borba00@gmail.com

Vivian Cristina de Melo

ORCID: https://orcid.org/0000-0002-6563-0105

Universidade Federal de Alagoas, Brasil

E-mail: vivian.melo@foufal.ufal.br

Wenys Cláudio Gomes da Silva

ORCID: https://orcid.org/0000-0002-9321-183X

Universidade Federal de Pernambuco, Brasil E-mail:wenys.claudio@ufpe.br

Fernanda Maria Matos Aragão de Souza

ORCID: https://orcid.org/0000-0002-4217-8013

Centro Universitário Tabosa de Almeida, Brasil E-mail: aragaofernanda22@gmail.com 


\author{
Camila Guerra Rêgo \\ ORCID: https://orcid.org/0000-0002-2410-319X \\ Faculdade de Odontologia do Recife, Brasil \\ E-mail: camilagr705@gmail.com \\ Matheus Vinicius Anunciação Neves \\ ORCID:: https://orcid.org/0000-0002-7878-0700 \\ Centro Universitário Tiradentes de Pernambuco, Brasil \\ E-mail: matheusviniciusneves10@outlook.com \\ Nathália Rayanne Torres \\ ORCID: https://orcid.org/0000-0002-0562-9268 \\ Centro Universitário Tiradentes de Pernambuco, Brasil \\ E-mail: nathaliasud@hotmail.com
}

\begin{abstract}
Resumo
Esse artigo buscou realizar um levantamento bibliográfico, de cunho narrativo, sobre o atendimento odontológico a gestante. Buscas virtuais foram realizadas nas bases de dados: PubMed, Cochrane Library, LILACS e na biblioteca virtual Scielo. Para isso, foi estabelecida a pergunta central: “Como é o atendimento odontológico as gestantes?” Foram incluídos trabalhos completos para avaliar o atendimento odontológico as gestantes. Por conseguinte, os artigos foram analisados criticamente nesta revisão. Este estudo visa analisar a literatura a cerca do atendimento odontológico a gestantes, devido à necessidade especial causada pelas mudanças no seu corpo. Verifica-se que muitos profissionais cirurgiões-dentistas recusam atendimento odontológico às gestantes por medo de causar aborto devido alterações fisiológicas que ocorrem durante o período gestacional, principalmente no período crítico da gestação (primeiro trimestre). Então optam por realizar os procedimentos cabíveis em outro período da gestação, por sentirem-se mais seguros para efetuar a prática. Com isso, o presente trabalho possui o objetivo de possibilitar esclarecimentos e recomendações sobre condutas clínicas por meio de evidências científicas relacionadas ao atendimento.
\end{abstract}

Palavras-chave: Gestante; Odontologia; Atendimento; Cuidado; Pré-natal.

\begin{abstract}
This article sought to carry out a bibliographical survey, of a narrative nature, on dental care for pregnant women. Virtual searches were performed in the databases: PubMed, Cochrane Library, LILACS and the Scielo virtual library. For this, the central question was established: "How is dental care for pregnant women?" Complete studies were included to assess the dental care provided to pregnant women. Therefore, the articles were critically analyzed in this review. This study aims to analyze the literature on dental care for pregnant women, due to the special need caused by changes in their bodies. It is found that many dental surgeons refuse dental care to pregnant women for fear of causing abortion due to physiological changes that occur during the gestational period, especially in the critical period of pregnancy (first trimester). So, they choose to perform the appropriate procedures at another period of pregnancy, because they feel safer to carry out the practice. [services, inevitably exposing its brands in social networks. The work evaluates the interaction of the organizations with the users in order to fulfill the mission of defending their brand in the face of judgments that may negatively expose the products and services of the brand that over the years struggles to survive the current economic situations of the country. Therefore, the present work aims to provide clarifications and recommendations on clinical behavior through scientific evidence related to dental care for pregnant women, enabling the establishment of an adequate treatment plan, a safe, humane and effective care.
\end{abstract}

Keywords: Pregnant women; Dentistry; Service; Caution; Prenatal care.

\title{
Resumen
}

Este artículo buscó realizar un relevamiento bibliográfico, de carácter narrativo, sobre la atención odontológica a la gestante. Se realizaron búsquedas virtuales en las bases de datos: PubMed, Cochrane Library, LILACS y la biblioteca virtual Scielo. Para ello, se estableció la pregunta central: "¿Cómo es el cuidado dental para la mujer embarazada?" Se incluyeron estudios completos para evaluar la atención dental brindada a las embarazadas. Por lo tanto, los artículos fueron analizados críticamente en esta revisión. Este estudio tiene como objetivo analizar la literatura sobre el cuidado dental de la mujer embarazada, debido a la especial necesidad que generan los cambios en su organismo. Se encuentra que muchos dentistas rechazan la atención dental a las embarazadas por temor a provocar un aborto debido a los cambios fisiológicos que ocurren durante el período gestacional, especialmente en el período crítico del embarazo (primer trimestre). Entonces, optan por realizar los procedimientos adecuados en otro período de embarazo, porque se sienten más seguras para realizar la práctica. Así, el presente trabajo tiene como objetivo aportar aclaraciones y recomendaciones sobre el comportamiento clínico por medio de la evidencia científica relacionada con la atención odontológica a la embarazada, que permita establecer un plan de tratamiento adecuado, una atención segura, humana y eficaz.

Palabras clave: Mujeres embarazadas; Odontología; Servicio; Precaución; Prenatal. 


\section{Introdução}

A manutenção e o acompanhamento de todas as fases da gestação, deve ser incluído nas atividades de rotina da Equipe de Saúde Bucal. Nenhuma necessidade de cuidados em saúde bucal das gestantes deve ser negligenciada pelo cirurgião-dentista, pelo medo de se colocar em risco a saúde do bebê. Manter o diálogo constante com o médico obstetra, aliado aos conhecimentos sobre os procedimentos seguros em cada fase da gestação, cada situação específica de gravidez dará ao cirurgião-dentista a segurança necessária para o atendimento e resolução das principais necessidades em saúde bucal das gestantes (Ribeiro, 2019).

O período gestacional é uma fase que requer uma atenção diferenciada pelas mudanças hormonais, físicas e psicológicas que ocorrem, necessitando de uma atenção odontológica especial, já que o meio bucal passa por desequilíbrios temporários que influenciam tanto mãe quanto concepto. Algumas das alterações que se pode observar, incluem: a erosão ácida do esmalte, doenças do periodonto, aumento de cárie dentária e perdas dentárias. Para tanto o profissional cirurgião-dentista, deve ter amplo conhecimento das alterações sistêmicas sobre a saúde das gestantes e promovera instrução sobre a saúde bucal, permitindo um amplo acesso aos serviços odontológicos e minimizando os riscos para a mãe e feto de uma condição bucal deficitária, além de tornar mais segura a relação entre paciente e profissional.

As transformações ocorridas no corpo da gestante culminam na criação de condições adversas, assim como alterações bucais, deixando-as mais suscetíveis a cárie e doença periodontal. Dentre as principais alterações bucais pode-se citar: náuseas, aumento da salivação e alterações no periodonto. A união dessas transformações aos hábitos de vida pode resultar no aparecimento ou agravamento de doenças periodontais (Nascimento et al., 2012).

O primeiro trimestre é o período mais critico da gestação, período da organogênese, pois ocorre o desenvolvimento dos órgãos do embrião. Esse período é considerado critico, devido ao maior risco de aborto espontâneo. Sendo assim, o cirurgiãodentista deve evitar procedimentos odontológicos nesse período, entretanto deve-se fazê-lo quando necessário (Garbin et al., 2011).

As gestantes precisam de atenção odontológica diferenciada visto que são consideradas paciente especial (Montadon et al., 2001). Bastiani et al. concluíram que 48,75\% das gestantes achavam que era normal desenvolver cárie dentária durante o período gestacional por causa da perda de mineral para os dentes dos bebês. Capucho et al. concluíram em seu estudo que $100 \%$ dos dentistas tinham algum tipo de dúvida sobre o atendimento odontológico em pacientes gestantes.

Um dos fatores que impossibilita as gestantes de procurem atendimento odontológico é o medo que existe em relação ao cirurgião dentista, principalmente neste período em que o corpo da mesma está sofrendo por tantas transformações e dentro dela há outro ser. É compreensível, contudo, é inadmissível que o dito medo faça com que a mesma não vá à procura de ajuda e até mesmo as consultas regulares. São nove meses, teoricamente, que a mãe carregará o filho e que permanecerá grávida, então, é completamente preocupante o não ir ao menos a uma consulta de rotina pois será neste momento que serão minimizados ou sanados alguns problemas bucais que atingem quando a mulher está gestante, de acordo obviamente com as restrições de cada uma, podendo ser preciso mais consultas. Como a exemplo de estudo, a gengivite grávidica, observada durante o período gestacional,cujo é causada pelo biofilme que se localiza próximo ou dentro do sulco gengival e está associada a fatores sistêmicos como as alterações hormonais (Silva et al., 2006, Xavier \& Xavier 2004).

A gengivite gravídica pode vir a ocorrer durante toda a gestação. Essa situação acarreta em uma resposta aumentada aos fatores locais e não é causada diretamente pela gravidez (Andrade 2006, Sonis et al., 1996). É caracterizada pela tumefação, vermelhidão e sangramento gengival (Sonis et al., 1996, Laskaris 2007).

Os sinais da gengivite generalizada podem ser mais frequentemente encontrados na gravidez principalmente relacionados a alterações nos hábitos de higiene, irritação local, impacção alimentar, má adaptação de restaurações, etc. (Tommasi,1989).

Outro problema que pode ser gerado é em relação aos dentes propriamente ditos, pode ser observada uma maior 
mobilidade dental devido à doença gengival associada a alterações minerais da lâmina dura (Xavier \& Xavier 2004). Essa maior mobilidade dental pode estar diretamente associada à redução na capacidade de substituição das fibras colágenas do periodonto (Bydlowski \& Douglas 1998).

Estudos feitos em meados de 2005 com um grupo de 100 gestantes foram observadas e descrita as as seguintes alterações; gengivite gravídica, granuloma gravídico, cloasma, telangiectasia facial, sialorréia, aumento na mobilidade dental e alterações na severidade das aftas. (Annan \& Nuamah 2005)

Segundo Oliveira et al. (2006), 37\% das gestantes examinadas em sua pesquisa apresentaram alterações bucais, dentre as quais a mais prevalente foi a gengivite $(91,9 \%)$, seguida do ressecamento dos lábios $(5,4 \%)$, fístula $(5,4 \%)$ e candidose $(0,37 \%)$.

Outro estudo, realizada por Gomes \& Paiva (2000) no qual foram examinados dois grupos de pacientes (gestantes e grupo controle de não gestantes), observou-se uma alta frequiência de gengivite em $100 \%$ das pacientes. O grupo das gestantes apresentou maior severidade de inflamação gengival em 49,5\% dos sítios periodontais examinados, como já era esperado, enquanto que as não gestantes apresentaram 36,6\% dos sítios periodontais examinados com inflamação gengival de maior severidade. Houve uma maior correlação entre inflamação gengival e higiene bucal no grupo controle do que no grupo gestante onde se propõe que o biofilme bacteriano é a responsável pelo início e manutenção da inflamação gengival, mas que algum outro fator pode atuar juntamente com este biofilme, acentuando o quadro inflamatório durante as fases de mudanças hormonais (como a gravidez).

Scavuzzi et al. (1998) enfatizou e evidenciou em uma pesquisa com 204 gestantes constatando que 98 delas (48\%) relataram ter sangramento gengival, sendo que dessas 69,4\% afirmaram que já o tinham desde antes da gravidez e $88,8 \%$ percebiam o sangramento quando escovavam os dentes ou usavam o fio dental. Segundo a percepção dos autores, pareceu que as gestantes acreditam ser normal o sangramento gengival, o que pode ter levado $52 \%$ das entrevistadas a não relatarem à presença desse sinal.

Então, com tantos estudos sobre a importância da consulta rotineira com o cirurgião dentista fica evidente e aclarado que os problemas podem ser evitados e surgem mais no período gestacional e esses não só acometem gengiva como muitas pessoas estão acostumadas a ouvir, pode também afetar a saúde sentia propriamente dita.

\section{Metodologia}

Refere-se a uma revisão integrativa de literatura, de caráter qualitativa. A revisão de literatura permite a busca aprofundada dentro de diversos autores e referenciais sobre um tema específico, nesse caso, as manifestações orais decorrentes da radioterapia de cabeça e pescoço (Pereira et al., 2018).

A fim de que haja direcionamento na pesquisa, delineou-se como questão norteadora: "Como é o atendimento odontológico as gestantes?"

\subsection{Busca Estratégica}

Para a construção desse artigo foi feito um levantamento bibliográfico de cunho narrativo, sobre o atendimento odontológico a gestante. Buscas virtuais foram realizadas nas bases de dados: PubMed, Cochrane Library, LILACS e na biblioteca virtual Scielo. Para isso, foi estabelecida a pergunta central: "Como é o atendimento odontológico as gestantes?" Foram incluídos trabalhos completos para avaliar o atendimento odontológico as gestantes. Os artigos foram coletados no período de janeiro a agosto de 2021 e contemplados entre os anos de 2000 a 2021. 


\subsection{Critérios de inclusão e exclusão}

Considerou-se como critério de inclusão os artigos completos disponíveis na íntegra nas bases de dados citadas, nos idiomas inglês e português e relacionados com o objetivo deste estudo.

Os critérios de exclusão foram artigos incompletos, duplicados, resenhas, estudos in vitro.

\subsection{Seleção dos estudos}

A estratégia de pesquisa baseou-se na leitura dos títulos para encontrar estudos que investigassem a temática da pesquisa. Caso atingisse esse primeiro objetivo, posteriormente, os resumos eram lidos e, persistindo na inclusão, era feita a leitura do artigo completo. Quando havia dúvida sobre a inclusão, o artigo era lido por outro autor e, a decisão de inclusão ou exclusão era tomada em consenso.

Na sequência metodológica foi realizada a busca e leitura na íntegra dos artigos pré-selecionados, os quais foram analisados para inclusão da amostra.

\section{Resultados e Discussão}

A gestação constitui um processo biológico natural da vida da mulher. Com tudo, nesse período ocorrem alterações fisiológicas no organismo da gestante, como alterações cardiovascular, hematológicas, respiratórias, renais, gastrointestinais e endócrinas, cujas consequências acabam refletindo na saúde bucal. Estudos demonstram a relação que existe entre a condição de saúde da cavidade oral com a saúde sistêmica, bem como as suas influências no desenvolvimento do bebê. Muitos mitos dissipados ao longo dos anos contribuem, para que mulheres nesta fase não procurem fazer o pré-natal odontológico, até mesmo em procedimentos emergenciais e, portanto, com indicação de intervenção imediata (Moretti, Garcia, Cruz, Rolim, \& Sakashita, 2017).

Além do medo e receio da gestante, existe também a insegurança por parte de muitos cirurgiões-dentistas em atender esse grupo de pacientes. Isso se dá por diversos motivos, dentre eles o início da carreira profissional, pois muitos não conseguem ter um ensino sobre esse tipo de atendimento, uma vez que são raras as universidades que possuem em sua grade curricular uma dedicação maior a gestantes, tornando uma falha na formação desses profissionais, acentuando ainda mais a sua insegurança no mercado (Assunção \& Miguel, 2015).

\subsection{Período gestacional e o atendimento odontológico}

Uma das maiores dúvidas de profissionais e gestantes é o período correto de realizar o atendimento. A maior parte dos procedimentos odontológicos em gestantes pode ser realizado em qualquer momento, porém alguns cuidados devem ser tomados como evitar sessões longas, acomodar a paciente na cadeira de modo que desconfortos sejam evitados e dar preferência a consultas em horário após a manhã, uma vez que nesse período a ânsia de vômito e risco de hipoglicemia é maior em gestantes (Bastiani et al., 2010).

Exodontias não complicadas, tratamentos periodontal e endodôntico, restaurações dentárias, instalação de próteses e outros tipos de procedimentos devem ser realizados com segurança, de preferência no segundo trimestre. Tratamentos seletivos como as reabilitações bucais extensas e as cirurgias mais invasivas podem ser programadas para o período de pós-parto.

O exame radiográfico deve ser realizado, quando realmente necessário, em qualquer trimestre da gestação; pois, desde que medidas protetoras sejam tomadas (uso de filmes ultrarápido e avental de chumbo) uma exposição radiográfica não afeta o desenvolvimento fetal. É necessário uma exposição de 5 rads para existir a possibilidade de má-formação ou aborto espontâneo, sendo que uma tomada radiográfica intrabucal equivale a 0,01 milirads de radiação, menos que a radiação cósmica adquirida diariamente. 
Ainda prevalece a crença de que gestantes não podem submeter-se à anestesia local, principalmente se os agentes anestésicos apresentarem vasoconstritor. No entanto, este tipo de anestesia é considerado seguro, desde que o profissional tenha conhecimento de quais substâncias medicamentosas utilizar. A solução anestésica local que apresenta maior segurança em gestantes é a lidocaína 2\% com adrenalina 1:100.000, respeitando-se o limite máximo de dois tubetes anestésicos (3,6ml) por sessão, procedendo sempre injeção lenta da solução.

Outro ponto bastante questionado na Odontologia é sobre a prescrição de fluoretos em gestantes. O conhecimento atual sobre o mecanismo de ação do fluoreto indica que seu efeito é predominantemente tópico, ocorrendo principalmente na interface placa/esmalte, através da remineralização de lesões de cárie iniciais e da redução da solubilidade do esmalte dentário. Portanto, a prescrição de medicamentos fluoretados no período pré-natal não traz nenhum beneficio que justifique sua indicação. No entanto há ressalvas, muitos problemas relacionados à cavidade bucal de gestantes poderiam ser evitados com cuidados básicos como orientações sobre higiene bucal e aplicação tópica de flúor, que podem ser feitos em qualquer época da gestação, sem ofertar nenhum perigo ao feto (Faria, 2010)

\subsection{Alterações bucais}

A gestação é acompanhada por diversas mudanças fisiológicas na mulher, necessitando assim de cuidados especiais temporários. Quando falamos da saúde bucal, o equilíbrio dela pode ser alterada por diversos motivos durante esse período, uma vez que a mulher estará mais sujeita a alterações hormonais, a uma dieta não balanceada, enjoos regulares e dificuldade de realizar a higienização bucal

(Silva, Couto, \& Conceição, 2020).

As principais alterações bucais que ocorrem durante o período gestacional são a cárie, gengivite e periodontite. No entanto, essas alterações não ocorreriam isoladamente apenas pelo fato da gravidez, mas sim por estarem atrelados a negligência na escovação e nos hábitos alimentares, como a maior ingestão de açúcar (Sampaio, 2019)

Vários fatores podem influenciar o surgimento da doença, o principal e mais negligenciado não só pela gestante mas por boa parte da população mundial é a deficiência na higiene oral, outro fator em são as doenças sistêmicas, como a diabetes, e a própria gestação em si, visto que nesse período, o organismo da mulher sofre muitas alterações morfológicas uma vez que as gestantes estão expostas a altas concentrações de hormônios esteroides produzidos pela placenta. Esses, possuem receptores no tecido gengival, acumulando-se o que constitui um importante fator para o crescimento bacteriano (Dourado, 2018).

O tumor gravídico também conhecido como granuloma piogênico (Xavier \& Xavier 2004, Fourniol 1998, Tommasi 1989, Neville et al., 2004), pode ser desenvolvido durante a gestação, e se caracteziza por um crescimento rápido (Orrico et al. 1987) podendo atingir dimensões que assustam o dentista e a paciente (Vieira \& Spalding 2006). Ocorre com mais incidência na maxila, com predileção pela face vestibular da região anterior, e com poucos casos que ultrapassam dois centímetros de diâmetro (Orrico et al. 1987). Na maioria dos casos regride após o fim do ciclo gestacional (Xavier \& Xavier 2004, Neville et al. 2004), sendo normalmente deixado sem tratamento até após o parto, a não ser em casos que se caracterizam como sintomáticos, de sangramento excessivo ou nos casos em que ocorre ulceração ou interferência na mastigação (Xavier \& Xavier 2004, Sonis et al. 1996). Nesses raros casos, deve ser removido cirurgicamente, envolvendo o tecido conjuntivo associado assim como qualquer outro fator etiológico presente (Xavier \& Xavier 2004, Sonis et al. 1996, Andrade 2006).

O granuloma gravídico apresenta as seguintes características clínicas: inflamação acentuada, gengiva marginal e papilar edematosa, mudança de coloração (vermelho intenso), aspecto liso e brilhante, consistência flácida e friável. Pode ser séssil ou pediculado, sangra com facilidade (Xavier \& Xavier 2004, Orrico et al. 1987) quando tocado (Orrico et al. 1987), mas geralmente é indolor. Andrade (2006) relata a aparência de amora devido ao aspecto granuloso e à cor vermelho escura. 
A gengivite observada durante a gravidez é causada pelo biofilme que se localiza próximo ou dentro do sulco gengival e está associada a fatores sistêmicos como alterações hormonais (Silva et al., 2006, Xavier \& Xavier 2004), ou seja, exacerbada pela intensa produção de hormônios sexuais femininos (Palmer \& Soory 2005). Normalmente recebe a denominação de gengivite gravídica (Palmer \& Soory 2005).

A gengivite gravídica pode ocorrer durante toda a gestação. Representa uma resposta aumentada aos fatores locais e não é causada diretamente pela gravidez (Andrade 2006, Sonis et al., 1996). É caracterizada pela tumefação, vermelhidão e sangramento gengival (Sonis et al., 1996, Laskaris 2007).

A manutenção da higiene bucal pode ajudar na prevenção ou redução da severidade dessas alterações inflamatórias gengivais mediadas pelo estrógeno e progesterona (Silva et al., 2006).

Pode ser observada maior mobilidade dental devido à doença gengival associada a alterações minerais da lâmina dura (Xavier \& Xavier 2004).

\subsection{Atenção básica na gravidez}

É durante a gestação a melhor época de orientar as mulheres sobre higiene bucal, a necessidade de controle do biofilme dentário e também de uma alimentação adequada, pois é nesse momento que elas estão mais propensas a adquirir novos conhecimentos, visando o bem estar da própria gestante e de seu futuro bebê, que podem levar a aquisição de novos hábitos saudáveis permanentes (Matsubara, 2017; Ribeiro, 2019).

Visando isso, o Sistema Único de Saúde (SUS), através da Estratégia da Saúde da Família (ESF), vem organizando Programas Nacionais de atenção à saúde da mulher, incluindo gestantes, por saber da importância em ofertar um serviço de reabilitação e recuperação da saúde bucal. Assim, faz parte da ESF realizar a assistência as gestantes de sua área, realizando o pré-natal (Lampert \& Bavaresco, 2017).

Dentro dos serviços prestado no período pré-natal, inclui o odontológico. Durante a gravidez, a mulher fica temporariamente em risco a alguns problemas bucais devido as alterações fisiológicas que podem ocorrer nesse período. Dessa forma, o pré-natal odontológico é de suma importância, visto que se torna uma medida de promoção da saúde, contribuindo não somente com a estabilidade da saúde bucal, como também na prevenção de futuros problemas sistêmicos (Nogueira, 2018).

\section{Conclusão}

É de importância imensurável que o cirurgião-dentista detenha do conhecimento e esteja apto para que o atendimento às gestantes seja realizado de forma adequada. Estas pacientes podem ser atendidas em qualquer trimestre de gestação, sendo o segundo, o mais seguro e confortável. No que se trata de terapêutica medicamentosa, o antibiótico de eleição é a penicilina e paracetamol, o analgésico de primeira escolha, deve-se evitar anti inflamatórios não esteróidais, e quando extremamente necessário, usa-se corticoides. Observa-se a necessidade de educação em saúde com mulheres gestantes como parte do tratamento odontológico para desmistificar crenças populares, possibilitando a inserção de novos hábitos que culminarão na promoção de saúde bucal da mulher e de seus filhos. Adicionalmente, a gestante é considerada uma paciente com necessidade especial então é de suma importância o cuidado diferenciado e individualizado devido às mudanças fisiológicas e psicológicas que ocorrem no período gestacional. Contudo, há uma necessidade do conhecimento por parte do Cirurgião-dentista para oferecer atendimento odontológico às gestantes de forma apropriada, diminuindo assim, o índice de atendimentos a gestantes recusados por esses profissionais ocasionado pelo medo de ambas as partes. Somado a isso, é importante incentivar as mulheres o cuidado odontológico durante a gestação, pelo menos uma vez a cada trimestre. Adicionalmente, verifica-se a necessidade de educação em saúde com mulheres gestantes (ações educativas e preventivas) como parte do tratamento odontológico para desmistificar 
crenças populares, é notória a carência, principalmente as que estão grávidas pela primeira vez e com isso possibilitar a inserção de novos hábitos que resultarão na promoção de saúde bucal da mulher e de seus filhos.

\section{Referências}

Andrade, E. D. (2006). Terapêutica Medicamentosa em Odontologia: Procedimentos clínicos e uso de medicamentos nas principais situações da prática odontológica. 2ed. Ed. Artes Médicas, p.129-130.

Assunção, A. C. \& Miguel, D. A. (2015). Assistência odontológica a gestante e lactantecom ênfase no tratamento endodôntico(Trabalho de conclusão de curso). Instituto de Estudos da Saúde Sérgio Feitosa, Belo Horizonte, MG, Brasil.

Bastiani, C., Cota, A. L. S., Provenzano, M. G. A., Fracasso, M. L. C., Honório, H. M. \& Rios, D. (2010). Conhecimento das gestantes sobre alterações bucais e tratamento odontológico durante a gravidez. Odontologia Clínico-Científica, 9 (2), 155-160.

Caneppele, T. M. F., Yamamoto, E. C., Souza, A. C., Valera, M. C. \& Araújo, M. A. M. (2011). Conhecimento dos cirurgiões-dentistas sobre o atendimento de pacientes especiais: hipertensos, diabéticos e gestantes. Journal of Biodentistry and Biomaterials, 1, 31-41.

Dourado, B. M. R. (2018). Doença periodontal em gestantes e repercussões gestacionais e ao recém-nascido (Dissertação de mestrado). Universidade Estadual Paulista Júlio de Mesquita Filho, Botucatu, SP, Brasil.

Faria, D. M. F. (2010). Atendimento odontológico às gestantes (Trabalho de conclusão de curso). Universidade Federal de Minas Gerais, Contagem, MG, Brasil.

Garbin, Cléa Adas Saliba. et al. (2011) Saúde coletiva: promoção de saúde bucal na gravidez. Rev. Odontol UNESP, Araraquara, v.40, n.4, jul-ago.

Lampert, L. \& Bavaresco, C. S. Atendimento Odontológico à gestante na atenção primária. RSC online, 6 (1), 81-95.

Laskaris G. (2007) Doenças da boca: texto e atlas. 2a ed. Porto Alegre: Artmed; p. 122-123; 232-233

Matsubara, A.S. \& Demetrio, A. T. W.; Atendimento odontológico às gestantes: Revisão de literatura. Revista Uningá Review, 29 (2).

Mesquita, Bruno da Silva. et al. (2013) Aspectos Norteadores da Atenção à Saúde Bucal de Gestantes da Estratégia Saúde da Família de João Pessoa, Brasil. Rev. Odontol. Bras. Central, v.22, n.60.

Montandon, Elisa Maria. et al. (2001) Hábitos dietéticos e de higiene bucal em mães no período gestacional. Jornal Brasileiro de Odontopediatria e Odontologia do Bebê,Curitiba, v.4, n.8, mar-abr.

Moretti, A. S., Garcia, V. B., Cruz, M. C. C. da, Rolim, V. C. de B., \& Sakashita, M. S. (2018). P oo9 - A importância do atendimento odontológico em gestantes. Archives of health investigation, 6.

Nascimento, É. P. et al. (2012). Gestantes frente ao tratamento odontológico. Rev. bras. Odontol., Rio de Janeiro. 69(1)

Palmer, R. \& Soory, M. (2005). Fatores Modificadores: Diabetes, Puberdade, Gravidez e Menopausa e Tabagismo. In: Lindhe, J., Karring, T. \& Lang, N. P. Tratado de Periodontia Clínica e Implantologia Oral. 4ed. Editora Guanabara Koogan. p. 181-2.

Ribeiro, L. P. (2019). Assistência odontológica na gestação (Trabalho de Conclusão de Curso). Centro Universitário do Planalto Central Apparecido dos Santos - UNICEPLAC, Brasília, DF, Brasil.

Sampaio, E. B. (2019). Percepção de gestantes acerca da saúde bucal na gravidez (Dissertação de Mestrado). Universidade Federal do Ceará, Fortaleza, CE, Brasil

Silva, L. G., Couto, L. S. \& Conceição, L. S. (2020). Cuidados odontológicos no pré-natal. Facit Business and Technology Journal, 2 (16), 174180 .

Xavier, H. S. \& Xavier, V. B. C. (2004). Alterações Decorrentes da Gravidez. In: Xavier, H. S. \& Xavier, V. B. C. Cuidados Odontológicos com a Gestante. Ed. Livraria Santos. p.5-7. 